\title{
Asymptotics of Young Diagrams and Hook Numbers
}

\author{
Amitai Regev* \\ Department of Theoretical Mathematics \\ The Weizmann Institute of Science \\ Rehovot 76100, Israel \\ and \\ Department of Mathematics \\ The Pennsylvania State University \\ University Park, PA 16802, U.S.A.
}

\section{Anatoly Vershik ${ }^{\dagger}$}

St. Petersburg branch of the Mathematics Institute

of the Russian Academy of Science

Fontanka 27

St. Petersburg, 191011 Russia

and

The Institute for Advanced Studies of the Hebrew University

Givat Ram

Jerusalem, Israel

Submitted: August 22, 1997; Accepted: September 21, 1997

\begin{abstract}
Asymptotic calculations are applied to study the degrees of certain sequences of characters of symmetric groups. Starting with a given partition $\mu$, we deduce several skew diagrams which are related to $\mu$. To each such skew diagram there corresponds the product of its hook numbers. By asymptotic methods we obtain some unexpected arithmetic properties between these products. The authors do not know "finite", nonasymptotic proofs of these results. The problem appeared in the study of the hook formula for various kinds of Young diagrams. The proofs are based on properties of shifted Schur functions, due to Okounkov and Olshanski. The theory of these functions arose from the asymptotic theory of Vershik and Kerov of the representations of the symmetric groups.
\end{abstract}

* Work partially supported by N.S.F. Grant No.DMS-94-01197.

$\dagger$ Partially supported by Grant INTAS 94-3420 and Russian Fund 96-01-00676 


\section{$\S 1$. Introduction and the main results}

Asymptotic calculations are applied to study the degrees of certain sequences of characters of symmetric groups $S_{n}, n \rightarrow \infty$. We obtain some unexpected arithmetic properties of the set of the hook numbers for some special families of (fixed) skew-Young diagrams (Theorem1.2). The problem appeared in the study of the hook formula for various kinds of Young diagrams. The proof of 1.2 is based on the properties of shifted Schur functions $[\underline{O k . O l}]$ which appeared in the asymptotic theory of the representation theory of the symmetric groups in $[\underline{V e r . K e r}]$. The authors do not know a "finite" proof of the theorem.

Given a partition $\mu$, we describe in [1.1] a construction of certain skew diagrams which are derived from $\mu$ : these are $S Q(\mu), S R(\mu), S R\left(\mu^{\prime}\right), R$ and $D_{\mu}$ below. Next, one fills these skew diagrams with their corresponding hook numbers $[\underline{M a c}]$ Theorem[1.2] which is the main result here, gives some divisibility properties of the products of these hook numbers.

We remark again that even though the statement of theorem[1.2] nothing to do with asymptotics, its proof does use asymptotic methods. It should be interesting to find an "asymptotic free" proof of Theorem[1.2]

We start with

1.1: A Construction: Given a partition (= diagram) $\mu$, let $D_{\mu}^{*}$ denote the double reflection of $\mu$. For example, if $\mu=(4,2,1)$ then

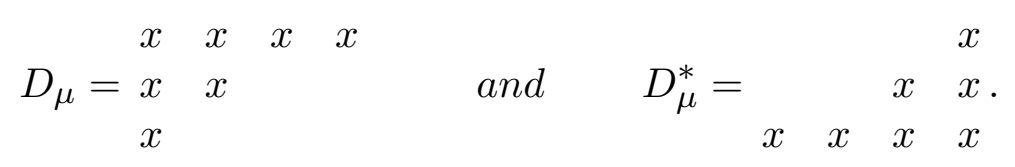

Recall that $\mu_{1}^{\prime}=\ell(\mu)$ is the number of nonzero parts of $\mu$. Complete $D_{\mu}^{*}$ to the $\mu_{1} \times \mu_{1}^{\prime}$ rectangle $R(\mu)$, then draw $D_{\mu}^{*}$ on top and on the left of $R$. Finally, erase the first $D_{\mu}^{*}$. Denote the resulting skew diagram by $S Q(\mu)$. For example, with $\mu=(4,2,1)$ we get 


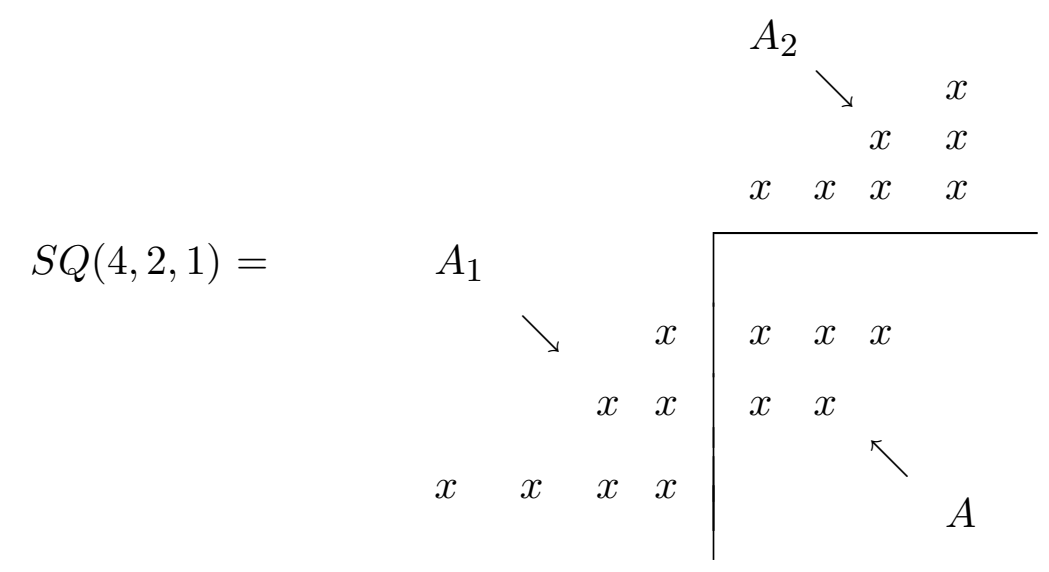

We subdivide $S Q(\mu)$ into the three areas $A, A_{1}$ and $A_{2}: A=R-D_{\mu}^{*}, A_{1}$ is the $D_{\mu}^{*}$ on the left of $R$ and $A_{2}$ is the $D_{\mu}^{*}$ on top of $R$. Denote $S R(\mu)=A_{1} \cup A$, the "shifted rectangle".

Clearly, $\left|A \cup A_{1}\right|=\left|A \cup A_{2}\right|=|R|,\left|A_{1}\right|=\left|A_{2}\right|=|\mu|$, so $|S Q(\mu)|=|R|+|\mu|$. Now, fill $S Q(\mu), S R(\mu), R$ and $\mu$ with their hook numbers. For example, when $\mu=(4,2,1)$

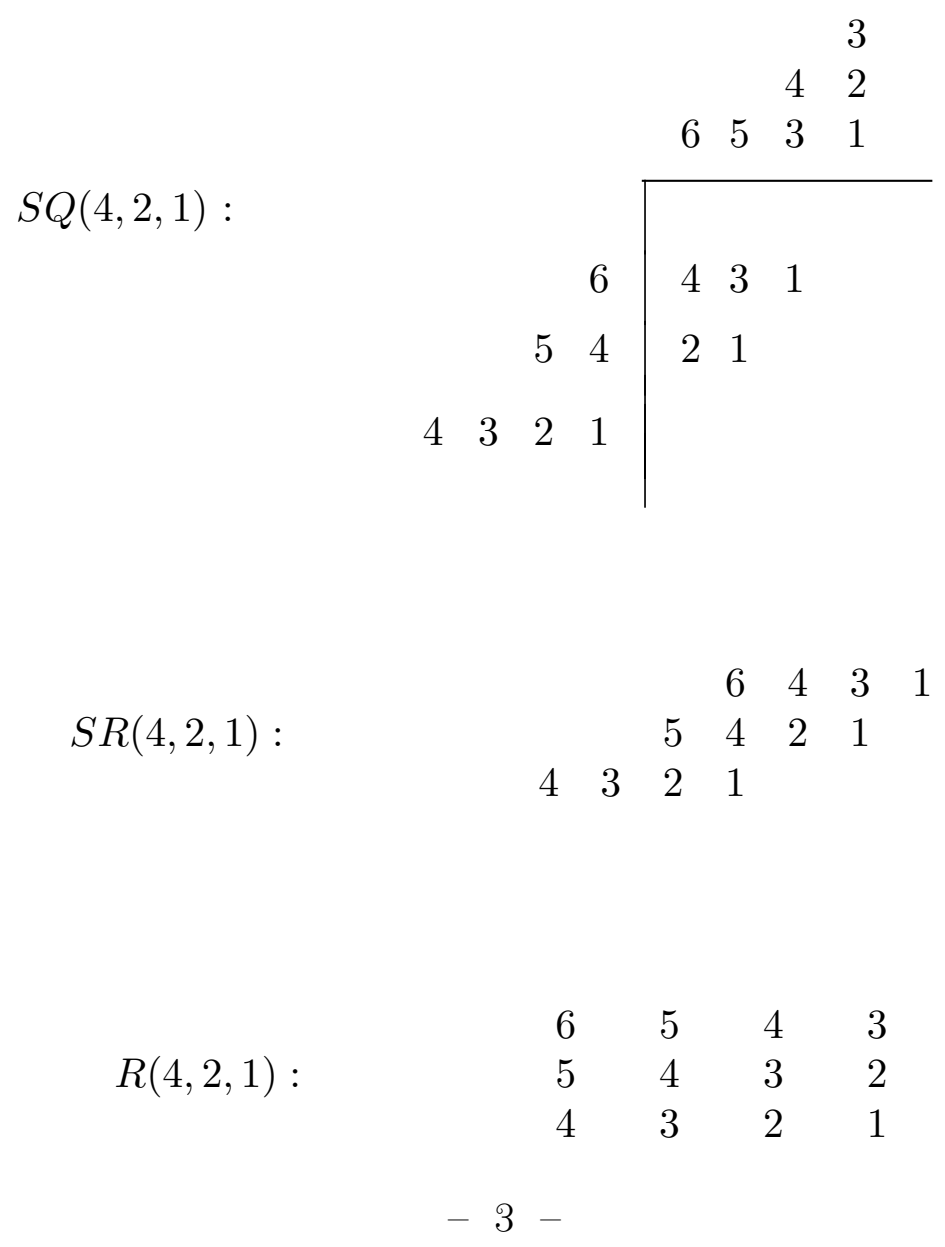


and

$$
\begin{array}{lllll} 
& 6 & 4 & 2 & 1 \\
(4,2,1): & 3 & 1 & & \\
& 1 & & &
\end{array}
$$

Thus, for example, $\prod_{x \in(4,2,1)} h(x)=1^{3} \cdot 2 \cdot 3 \cdot 4 \cdot 6=144$.

Note that the hook numbers in $S R(\mu)$ are the same as those in the area $A_{1} \cup A$ of $S Q(\mu)$.

As usual, $\mu_{1}^{\prime}=\ell(\mu)$ is the number of nonzero parts of $\mu$. Recall that $s_{\mu}\left(x_{1}, x_{2}, \cdots\right)$ is the corresponding Schur function, and $s_{\mu} \underbrace{(1, \cdots, 1)}_{\mu_{1}^{\prime}}$ is the number of (semi-standard, i.e. rows weakly and column strictly increasing) tableaux of shape $\mu$, filled with elements from $\left\{1,2, \cdots, \mu_{1}^{\prime}\right\}[\underline{M a c}]$ Similarly for $s_{\mu^{\prime}} \underbrace{(1, \cdots, 1)}_{\mu_{1}}$.

1.2 Theorem: Let $\mu$ be a partition. With the above construction of $S Q(\mu)=A \cup A_{1} \cup A_{2}$ and $R$, we have

$$
\left(\prod_{x \in R} h(x)\right) /\left(\prod_{x \in A_{1} \cup A} h(x)\right)=s_{\mu}(\underbrace{1, \cdots, 1}_{\mu_{1}^{\prime}}) .
$$

In particular, $\prod_{x \in A_{1} \cup A} h(x)$ divides $\prod_{x \in R} h(x)$. [Note that $A \cup A_{1} \subset S Q(\mu)$, and for $x \in A_{1} \cup A, h(x)$ is the corresponding hook number in $x \in S Q(\mu)$ ].

(1') Similarly,

$$
\left(\prod_{x \in R} h(x)\right) /\left(\prod_{x \in A_{2} \cup A} h(x)\right)=s_{\mu^{\prime}}(\underbrace{1, \cdots, 1}_{\mu_{1}}) .
$$

$$
\prod_{x \in S Q(\mu)} h(x)=\left(\prod_{x \in R} h(x)\right) \cdot\left(\prod_{x \in \mu} h(x)\right) .
$$


We conjecture that a statement much stronger than $[\underline{1.2 .2}]$ holds, namely: the two multisets $\{h(x) \mid x \in S Q(\mu)\}$ and $\{h(x) \mid x \in R\} \cup\{h(x) \mid x \in \mu\}$ are equal.

Theorem[1.2.1] is an obvious consequence of the following "asymptotic" theorem.

1.3. Theorem: Let $\mu=\left(\mu_{1}, \cdots, \mu_{k}\right)$, be a partition. Let $n=k \ell$, $\mu_{1} \leq \ell \rightarrow \infty$, and denote $\lambda=\lambda(\ell)=\left(\ell^{k}\right)$. Then

$$
\lim _{\ell \rightarrow \infty} \frac{d_{\lambda / \mu}}{d_{\lambda}}=\left(\frac{1}{k}\right)^{|\mu|} \cdot s_{\mu}(\underbrace{1, \cdots, 1}_{k})
$$

and

$$
\lim _{\ell \rightarrow \infty} \frac{d_{\lambda / \mu}}{d_{\lambda}}=\left(\frac{1}{k}\right)^{|\mu|} \cdot\left(\prod_{x \in R\left(\mu_{1}, \mu_{1}^{\prime}\right)} h(x)\right) /\left(\prod_{x \in A_{1} \cup A} h(x)\right)
$$

Theorem $\left[\underline{1.2 .1^{\prime}}\right]$ follows from $[\underline{1.2 .1}]$ by conjugation.

Theorem[1.2.2] is a consequence of the following "asymptotic" theorem

1.4. Theorem: Let $\mu$ be a fixed partition. Let $\mu_{1} \leq \ell \rightarrow \infty$, $\mu_{1}^{\prime} \leq m \rightarrow \infty, n=\ell m$ and $\lambda=\lambda(\ell, m)=\left(\ell^{m}\right)$. Then

$$
\begin{gathered}
\lim _{\ell, m \rightarrow \infty} \frac{d_{\lambda / \mu}}{d_{\lambda}}=\frac{1}{\prod_{x \in \mu} h(x)} \\
\lim _{\ell, m \rightarrow \infty} \frac{d_{\lambda / \mu}}{d_{\lambda}}=\left(\prod_{x \in R} h(x)\right) /\left(\prod_{x \in S Q(\mu)} h(x)\right) .
\end{gathered}
$$

In this note we apply the following main tools:

a) The theory of symmetric functions $[\underline{M a c}$,$] . In particular, we apply the hook$ formula

$$
\begin{aligned}
d_{\lambda}= & \frac{|\lambda| !}{\prod_{x \in \lambda} h(x)} \\
& -5-
\end{aligned}
$$


and I.3, Example 4, page 45 in $[\underline{M a c}$,$] .$

b) The Okounkov-Olshanski $[\underline{\mathrm{Ok} . \mathrm{Ol}}]$ theory of "shifted symmetric functions". In particular, we apply formula (0.14) of $[\underline{O k . O l}]$ Let $\mu \vdash k, \lambda \vdash n, k \leq n, \mu \subset \lambda$, then

$$
\frac{d_{\lambda / \mu}}{d_{\lambda}}=\frac{s_{\mu}^{*}(\lambda)}{n(n-1) \cdots(n-k+1)} .
$$

Here $s_{\mu}^{*}(x)$ is the "shifted Schur function" $[\underline{O k . O l}]$; one of its key properties is that $s_{\mu}^{*}(x)=s_{\mu}(x)+$ lower terms, where $s_{\mu}(x)$ is the ordinary Schur function.

We remark that the paper $[\underline{O k . O l}]$ was influenced by the work of Vershik and Kerov on the asymptotic theory of the representations of the symmetric groups. See for example[ $[\underline{V e r} . \mathrm{Ker}]$, in which the characters of the infinite symmetric group are found from limits involving ordinary Schur functions. See also the introduction of $[\underline{O K . O l}]$

$\S 2$. Here we prove theorem [1.3] which, as noted before, implies [1.2.1] (and $\left.1.2 .1^{\prime}\right)$.

\subsection{The proof of theorem $[\underline{1.3}]$.}

$$
\frac{d_{\lambda(\ell) / \mu}}{d_{\lambda(\ell)}}=\frac{s_{\mu}^{*}\left(\lambda_{1}(\ell), \cdots, \lambda_{k}(\ell)\right)}{n(n-1) \cdots(n-|\mu|+1)},
$$

where $n=|\lambda|=k \ell$. Since $\ell \rightarrow \infty, n(n-1) \cdots(n-|\mu|+1) \simeq(k \ell)^{|\mu|}$. Also,

$$
s_{\mu}^{*}(\lambda)=s_{\mu}(\lambda)+(\text { lower terms in } n) \text {, }
$$

hence

$$
s_{\mu}^{*}(\lambda) \simeq s_{\mu}(\lambda)=s_{\mu} \underbrace{(\ell, \cdots, \ell)}_{k} .
$$

Recall that for two sequences $a_{n}, b_{n}$ of real numbers, $a_{n} \simeq b_{n}$ means that $\lim _{n \rightarrow \infty} \frac{a_{n}}{b_{n}}=1$. 
Since $s_{\mu}(x)$ is homogeneous of degree $|\mu|$,

$$
s_{\mu}(\lambda)=\ell^{|\mu|} \cdot s_{\mu}(\underbrace{1, \cdots, 1}_{k}) .
$$

The proof now follows easily.

2.2. The proof of theorem $[\underline{1.3 . b}]$ Since $\lambda$ is a rectangle, hence $d_{\lambda / \mu}=d_{\eta}$, where $\eta$ is the double reflection of $\lambda / \mu$. Denote by $\tilde{\mu}=D_{\mu}^{*}$ the double reflection of $\mu$. Thus

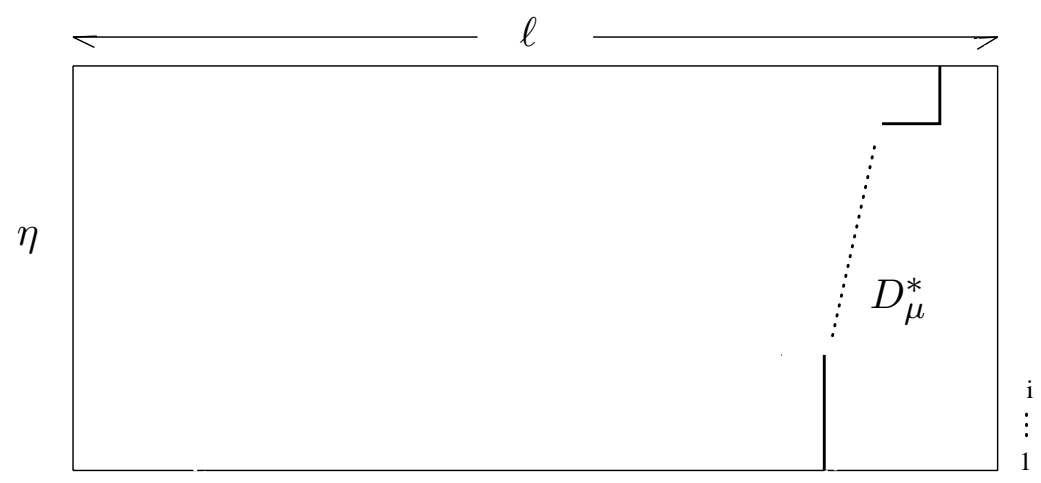

To calculate $d_{\lambda}$ and $d_{\eta}$ by the hook formula, fill $\lambda=\lambda(\ell)$ and $\eta$ with their respective hook numbers. In both, examine the $i^{\text {th }}$ row from the bottom - with their respective hook numbers. Divide $\eta$ into $B_{1}$ and $B_{2}$ as follows:

Notice that $B_{1}=S R(\mu)$ of 1.1. Note also that the hook numbers in $B_{1}$ are those in $S R(\mu)$, and they are independent of $\ell$.

Examine the hook numbers in $B_{2}$. In the $i^{\text {th }}$ row (from bottom), these are $\mu_{1}+i, \mu_{1}+i+1, \cdots, \ell+i-1-\mu_{i}$, consecutive integers.

We also divide $\lambda(\ell)$ into two rectangles:

Again, the hook numbers in $R_{1}$ are independent of $\ell$, and those in the $i^{\text {th }}$ row (from bottom) of $R_{2}$ are $\mu_{1}+i, \mu_{1}+i+1, \cdots, \ell+i-1$, again consecutive integers. 

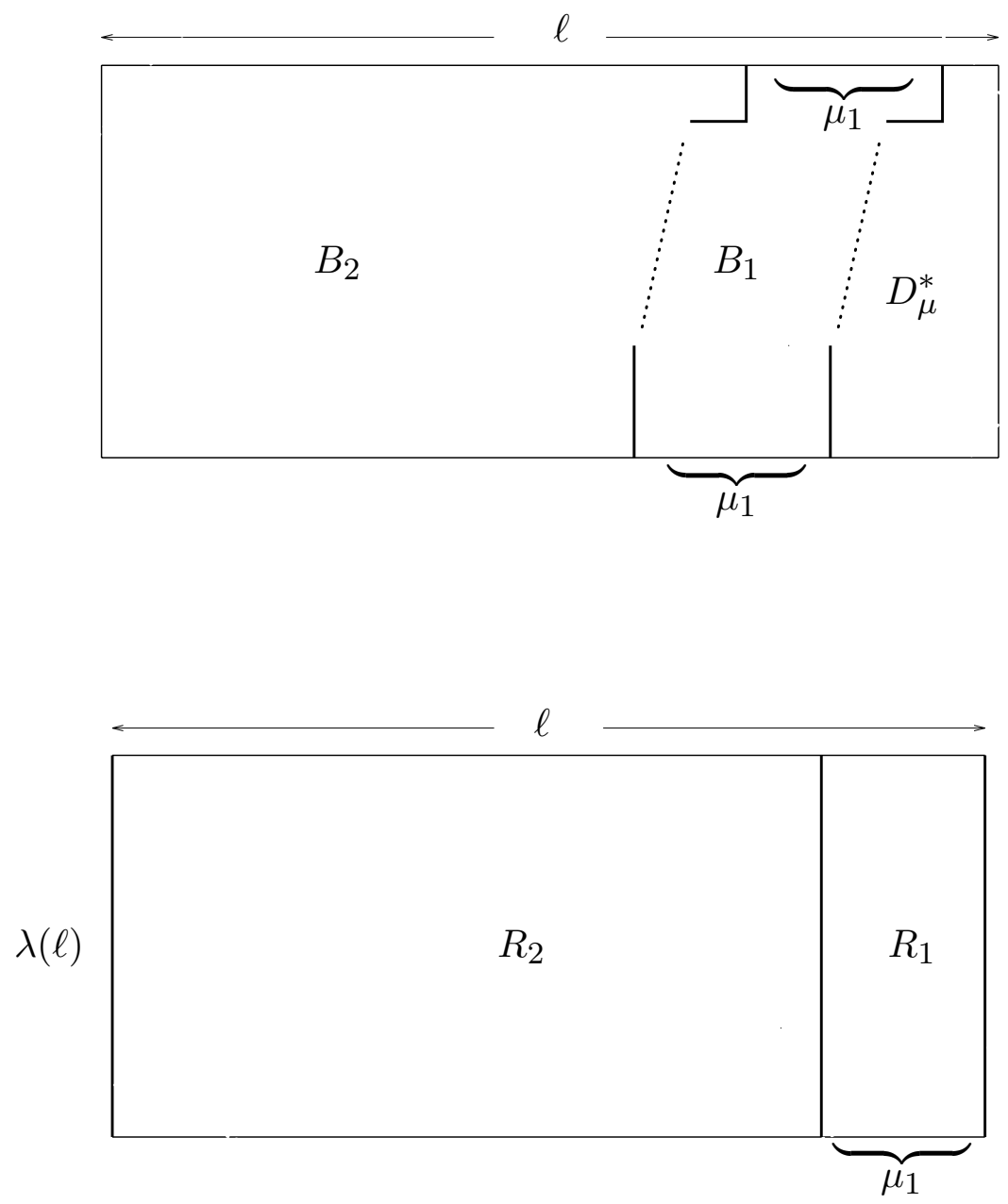

By the "hook" formula, the left hand side of 1.3.b is

$$
\begin{gathered}
\frac{d_{\lambda(\ell) / \mu}}{d_{\lambda(\ell)}}=\frac{d_{\eta}}{d_{\lambda(\ell)}}=\left[\frac{(n-|\mu|) !}{\prod_{x \in \eta} h(x)}\right] /\left[\frac{n !}{\prod_{x \in \lambda(\ell)} h(x)}\right] \\
=\frac{(n-|\mu|) !}{n !} \cdot\left[\frac{\prod_{x \in \lambda(\ell)} h(x)}{\prod_{x \in \eta} h(x)}\right]
\end{gathered}
$$

where $n=k \ell$. Since $\ell \rightarrow \infty$,

$$
\frac{(n-|\mu|) !}{n !} \simeq\left(\frac{1}{n}\right)^{|\mu|}=\left(\frac{1}{k \ell}\right)^{|\mu|}
$$

Now

$$
\frac{\prod_{x \in \lambda(\ell)} h(x)}{\prod_{x \in \eta} h(x)}=\left[\frac{\prod_{x \in R_{1}} h(x)}{\prod_{x \in B_{1}} h(x)}\right] \cdot\left[\frac{\prod_{x \in R_{2}} h(x)}{\prod_{x \in B_{2}} h(x)}\right]=\alpha \cdot \beta .
$$


Note that the right hand side of $1.3 . \mathrm{b}$ is $\left(\frac{1}{k}\right)^{|\mu|} \cdot \alpha$.

We calculate $\beta$ :

$$
\begin{gathered}
\prod_{x \in R_{2}} h(x)=\prod_{i=1}^{\mu_{1}^{\prime}}\left[\left(\mu_{1}+i\right)\left(\mu_{1}+i+1\right) \cdots(\ell+i-1)\right], \\
\prod_{x \in B_{2}} h(x)=\prod_{i=1}^{\mu_{1}^{\prime}}\left[\left(\mu_{1}+i\right)\left(\mu_{1}+i+1\right) \cdots\left(\ell+i-1-\mu_{i}\right)\right],
\end{gathered}
$$

thus

$$
\beta=\prod_{i=1}^{\mu_{1}^{\prime}}\left[\left(\ell+i-\mu_{i}\right)\left(\ell+i-\mu_{i}+1\right) \cdots(\ell+i-1)\right] \simeq \ell^{|\mu|},
$$

(since $\ell \rightarrow \infty)$.

Hence,

$$
\lim _{\ell \rightarrow \infty} \frac{d_{\lambda(\ell) / \mu}}{d_{\lambda(\ell)}}=\left(\frac{1}{k}\right)^{|\mu|} \cdot \alpha
$$

and the proof is complete.

$\S$ 3. Here we prove theorem 1.4 which, as noted before, implies theorem 1.2.2.

3.1. The proof of 1.4.a: Let $\lambda=\lambda(\ell, m)=\left(\ell^{m}\right), \ell, m \rightarrow \infty$. We show first that $s_{\mu}^{*}(\lambda) \simeq s_{\mu}(\lambda)$, as follows: By [Ok.Ol.(0.9)],

$$
\begin{aligned}
e_{r}^{*}(\lambda) & =\sum_{i \leq i_{1}<\cdots<i_{r} \leq m}(\ell+r-1)(\ell+r-2) \cdots \ell= \\
& =(\ell+r-1)(\ell+r-2) \cdots \ell \cdot\left(\begin{array}{c}
m \\
r
\end{array}\right) \simeq \frac{\ell^{r} m^{r}}{r !} .
\end{aligned}
$$

Similarly, $e_{r}(\lambda) \simeq \frac{\ell^{r} m^{r}}{r !}$

Let $\emptyset$ be given as in [Ok.Ol.§13]. By [Ok.Ol.(13.8)] it easily follows that for any $u$ and $r$,

$$
\begin{aligned}
\emptyset^{-u} e_{r}^{*}(\lambda) & \simeq e_{r}^{*}(\lambda) \simeq e_{r}(\lambda) \\
& -9-
\end{aligned}
$$


Applying the Jacobi Trudi formulas for $s_{\mu}(\lambda)-([\underline{M a c},] \mathrm{I},(3.5)$, page 41] and for $s_{\mu}^{*}(\lambda)[\underline{O k . O l}(13.10)]$ that $s_{\mu}^{*}(\lambda) \simeq s_{\mu}(\lambda)$. Now in $[\underline{2.1}$,$] , here$

$$
\frac{d_{\lambda(\ell, m) / \mu}}{d_{\lambda(\ell, m)}}=\frac{s_{\mu}^{*}\left(\lambda_{1}(\ell, m), \cdots, \lambda_{m+k}(\ell, m)\right)}{n(n-1) \cdots(n-|\mu|+1)}
$$

where

$$
n=\ell m
$$

Here

$$
s_{\mu}^{*}(\lambda(\ell, m)) \simeq s_{\mu}(\lambda(\ell, m))=\ell^{|\mu|} s_{\mu}(\underbrace{1, \cdots, 1}_{m})
$$

Thus

$$
\frac{d_{\lambda(\ell, m) / \mu}}{d_{\lambda(\ell, m)}} \simeq\left(\frac{1}{n}\right)^{|\mu|} \cdot s_{\mu}(\underbrace{1, \cdots, 1}_{m})=\left(\frac{1}{m}\right)^{|\mu|} \cdot \prod_{x \in \mu} \frac{m+c(x)}{h(x)}
$$

([[ $\underline{M a c}]$, pg. 45, Ex 4]) where $c(x)$ is the content of $x \in \mu$. Since $m \rightarrow \infty, m+$ $c(x) \simeq m$ for all $x \in \mu$, and the proof follows.

3.2. The proof of $[\underline{1.4 b}] \quad$ Choose $\ell, m$ large so that $\mu \subset \lambda(\ell, m)$. Let $\eta$ be the double reflection of $\lambda(\ell, m) / \mu$, so $d_{\lambda(\ell, m) / \mu}=d_{\eta}$, then calculate $d_{\eta}$ by the hook formula. To analyze the hook numbers in $\eta$, we subdivide $\eta$ into the areas $A_{1, \eta}, \cdots, A_{4, \eta}$ as shown below:

i.e., $D_{\mu}^{*}$ is drawn at the bottom-right of the $\ell \times m$ rectangle. We then follow [1.1] and construct $A_{4, \eta}=S Q(\mu)$. Now $A_{1, \eta}$ is the $\left(\ell-\mu_{1}\right) \times\left(m-\mu_{1}^{\prime}\right)$ rectangle, and this determines $A_{2, \eta}$ and $A_{3, \eta}$.

We also split the $\ell \times m$ rectangle $\lambda=\lambda(\ell, m)$ accordingly:

Since $\lambda(\ell, m) \vdash \ell m$ and $\eta \vdash \ell m-|\mu|$,

$$
\frac{d_{\eta}}{d_{\lambda(\ell, m)}} \simeq\left(\frac{1}{\ell m}\right)^{|\mu|} \cdot \frac{\prod_{x \in \lambda(\ell, m)} h_{\lambda(\ell, m)}(x)}{\prod_{x \in \eta} h_{\eta}(x)} .
$$



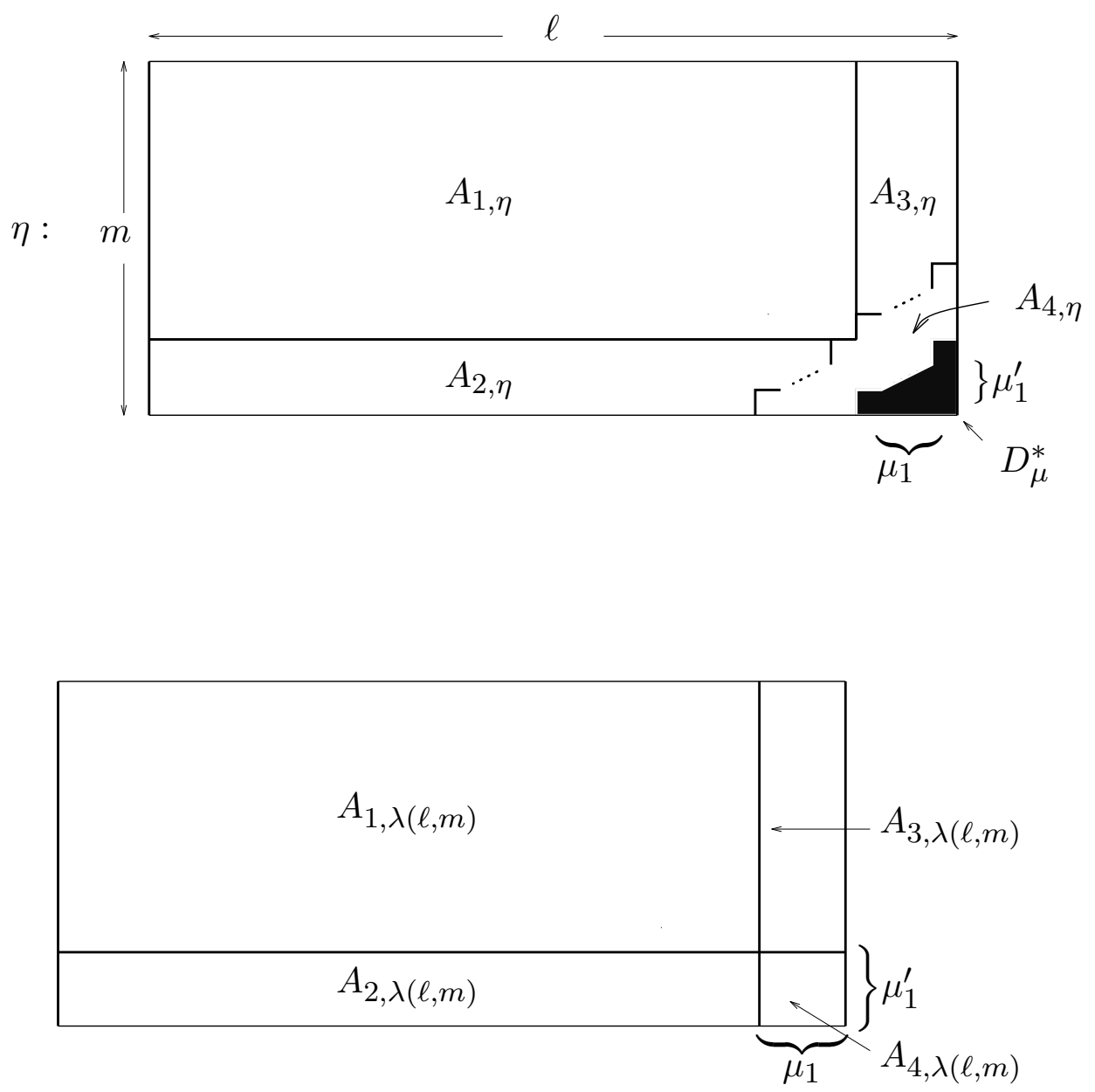

Now, $h_{\lambda(\ell, m)}(x)=h_{\eta}(x)$ for $x \in A_{1, \eta}=A_{1, \lambda(\ell, m)}$. As in 2.3

$$
\frac{\prod_{x \in A_{2, \lambda(\ell, m)}} h_{\lambda(\ell, m)}(x)}{\prod_{x \in A_{2, \eta}} h_{\eta}(x)} \simeq \ell^{|\mu|} .
$$

Similarly (or, by conjugation),

$$
\frac{\prod_{x \in A_{3, \lambda}} h_{\lambda(\ell, m)}(x)}{\prod_{x \in A_{3, \eta}} h_{\eta}(x)}=m^{|\mu|} .
$$


After cancellations we have

$$
\frac{d_{\eta}}{d_{\lambda}} \simeq \frac{\prod_{x \in A_{4, \lambda}} h_{\lambda(\ell, m)}(x)}{\prod_{x \in A_{4, \eta}} h_{\eta}(x)}=\frac{\prod_{x \in R\left(\mu_{1}, \mu_{1}^{\prime}\right)} h(x)}{\prod_{x \in S Q(\mu)} h(x)}
$$

and the proof is complete.

\section{References}

[Ok.Ol] Okounkov A. and Olshanski G., Shifted Schur functions, preprint.

[Mac] Macdonald I.G., Symmetric functions and Hall polynomials, Oxford University Press, 2nd edition 1995.

[Ver.Ker] Vershik A.M. and Kerov, S.V., Asymptotic Theory of characters of the symmetric group, Funct. Anal. Appl. 15 (1981) 246-255.

email addresses: regev@wisdom.weizmann.ac.il, vershik@pdmi.ras.ru 